\title{
Choice Behavior Guided by Learned, But Not Innate, Taste Aversion Recruits the Orbitofrontal Cortex
}

\author{
○Leticia Ramírez-Lugo, @Ana Peñas-Rincón, Sandybel Ángeles-Durán, and ĐFrancisco Sotres-Bayon \\ Instituto de Fisiología Celular-Neurociencias, Universidad Nacional Autónoma de México, 04510 Ciudad de México, México
}

The ability to select an appropriate behavioral response guided by previous emotional experiences is critical for survival. Although much is known about brain mechanisms underlying emotional associations, little is known about how these associations guide behavior when several choices are available. To address this, we performed local pharmacological inactivations of several cortical regions before retrieval of an aversive memory in choice-based versus no-choice-based conditioned taste aversion (CTA) tasks in rats. Interestingly, we found that inactivation of the orbitofrontal cortex $(\mathrm{OFC})$, but not the dorsal or ventral medial prefrontal cortices, blocked retrieval of choice CTA. However, OFC inactivation left retrieval of no-choice CTA intact, suggesting its role in guiding choice, but not in retrieval of CTA memory. Consistently, OFC activity increased in the choice condition compared with no-choice, as measured with c-Fos immunolabeling. Notably, OFC inactivation did not affect choice behavior when it was guided by innate taste aversion. Consistent with an anterior insular cortex (AIC) involvement in storing taste memories, we found that AIC inactivation impaired retrieval of both choice and no-choice CTA. Therefore, this study provides evidence for OFC's role in guiding choice behavior and shows that this is dissociable from AIC-dependent taste aversion memory. Together, our results suggest that $\mathrm{OFC}$ is required and recruited to guide choice selection between options of taste associations relayed from AIC.

Key words: conditioning; decision; gustatory; inactivation; insular; prefontal

Significance Statement

Survival and mental health depend on being able to choose stimuli not associated with danger. This is particularly important when danger is associated with stimuli that we ingest. Although much is known about the brain mechanisms that underlie associations with dangerous taste stimuli, very little is known about how these stored emotional associations guide behavior when it involves choice. By combining pharmacological and immunohistochemistry tools with taste-guided tasks, our study provides evidence for the key role of orbitofrontal cortex activity in choice behavior and shows that this is dissociable from the adjacent insular cortex-dependent taste aversion memory. Understanding the brain mechanisms that underlie the impact that emotional associations have on survival choice behaviors may lead to better treatments for mental disorders characterized by emotional decisionmaking deficits.

\section{Introduction}

Recollection of previous experiences charged with emotional content can have a profound impact on decisions that are funda-

\footnotetext{
Received March 10, 2016; revised Aug. 23, 2016; accepted Aug. 25, 2016

Author contributions: L.R.-L. and F.S.-B. designed research; L.R.-L., A.P.-R., and S.Á.-D. performed research; L.R.-L., A.P.-R., and F.S.-B. analyzed data; F.S.-B. wrote the paper.

This work was supported by the Consejo Nacional de Ciencia y Tecnología (Grant 176639), the Dirección General de Asuntos del Personal Académico de la Universidad Nacional Autónoma de México (Grants IA200313 and IA200715), and the International Brain Research Organization (Return Home fellowship) to F.S.-B. We thankJ. OrtizAlegría and R. Martinez-Gómez for technical assistance. We also thank G.J. Quirk and C. Bravo-Rivera for helpful comments on this manuscript.

The authors declare no competing financial interests.

Correspondence should be addressed to Francisco Sotres-Bayon, Ph.D., Instituto de Fisiología CelularNeurociencias, Universidad Nacional Autónoma de México, 04510 Ciudad de México, México. E-mail: sotres@ifc.unam.mx.

DOI:10.1523/JNEUROSCI.0796-16.2016

Copyright $\odot 2016$ the authors $\quad 0270-6474 / 16 / 3610574-10 \$ 15.00 / 0$
}

mental for survival (Rangel et al., 2008). Deficits in such emotional or value-based decision-making ability characterize several psychiatric disorders (Paulus, 2007). Through associative mechanisms, environmental stimuli can acquire emotional valence (negative or positive), which in turn serve as motivation to select the appropriate action to take (avoid or approach) (Namburi et al., 2016). The influence of these emotional associations on action selection can be studied using conditioning (Rangel et al., 2008). Most often, they have been studied by examining their effect on instrumental appetitive responses (Holmes et al., 2010), but little research has been devoted to examining their effect on simple aversive learned responses. During aversive conditioning, a neutral stimulus acquires a negative motivational value when it is paired with a negative consequence. This association forms an aversive memory that, when retrieved, evokes stimulus-triggered defensive responses that include (depending on the conditioning 
method) freezing, avoidance (Bravo-Rivera et al., 2014) and disgust (Bures et al., 1998). Much effort has been directed toward understanding the associative brain mechanisms involved in aversive processing [e.g., taste aversion (Bermúdez-Rattoni, 2004) and threat conditioning (LeDoux, 2000; Maren and Quirk, 2004)], but rather less is known about the process by which the resultant aversive memory influences the actions that lead to avoid the stimuli.

Extensive research using taste-digestive malaise (conditioned taste aversion, CTA) and tone-shock (auditory threat conditioning) associations have delineated the pathways that are shared in processing aversive memories. Both of these types of amygdaladependent aversive conditioning (LeDoux et al., 1990; Gallo et al., 1992), involve the recruitment of primary sensory cortex associated with the conditioned stimuli [i.e., taste cortex for CTA (Gallo et al., 1992) and auditory cortex for auditory threat conditioning (Quirk et al., 1997)]. Further cortical processing of aversive memories, independently of the aversive conditioning type, involves the medial prefrontal cortex (mPFC) (Hernádi et al., 2000; Mickley et al., 2005; Knapska and Maren, 2009; Lin et al., 2010; Sierra-Mercado et al., 2011). Notably, dorsal mPFC has been shown to be involved in the expression of aversive memory (Corcoran and Quirk, 2007; Bissière et al., 2008; Marotta et al., 2014; Gonzalez et al., 2015), whereas ventral mPFC is involved in extinction of aversive memory (Milad and Quirk, 2002; Akirav et al., 2006; Sotres-Bayon et al., 2009; Xin et al., 2014). These findings have led to the notion that coordinated activity between different cortical regions is involved in simple retrieval of aversive memories (Bermúdez-Rattoni, 2004; Sotres-Bayon and Quirk, 2010). However, it is not clear whether the same cortical regions are required when the individual is challenged to use the retrieved aversive memory to choose between options presented simultaneously.

To evaluate which cortical areas are critical for choice behavior guided by an aversive memory, we developed a modified version of the standard CTA paradigm. Execution of an avoidance response to a taste stimulus during CTA is a behavior more amenable to studying the neurobiology of choice than stimulustriggered reaction (freezing) evoked during retrieval of cued threat conditioning. We tested aversive memory retrieval in a choice-based versus no-choice-based CTA task. In the standard CTA task, rats learn to avoid a single bottle containing the aversive taste solution without a choice (Bures et al., 1998). To evaluate choice behavior, we developed a modified version of the CTA task that involves learning to choose actively between bottles presented simultaneously containing an aversive taste solution and safe water. Our findings indicate that the orbitofrontal cortex (OFC), rather than the primary taste cortex or mPFC, guides choice behavior for learned, but not innate, taste-aversive memories.

\section{Materials and Methods}

\section{Subjects}

Male Wistar rats (280-300 g; Instituto de Fisiología Celular breeding colony) were housed in polyethylene cages and maintained on a standard $12 \mathrm{~h}$ light/dark schedule with ad libitum access to standard laboratory rat chow and water. Rats were deprived of water for $24 \mathrm{~h}$ before the start of all behavioral manipulations to motivate them to consume water from the bottle(s) presented. Rats were accustomed to drink water in 2 independent sessions separated by $6 \mathrm{~h}$. The first consumption session was in the morning and the second was in the afternoon. All manipulations and behavioral procedures were performed during the light phase. Animals were housed individually in a temperature-controlled environment $\left(24^{\circ} \mathrm{C}\right)$ for at least $4 \mathrm{~d}$ before surgery and were handled daily to diminish stress responses. All procedures were approved by the Institutional Animal Care and Use Committee of the Universidad Nacional Autónoma de México in compliance with the National Ministry of Health guidelines for the care of laboratory animals.

\section{Surgery}

Rats were anesthetized (mixture of ketamine $90 \mathrm{mg} / \mathrm{kg}$ and xylazine $10 \mathrm{mg} / \mathrm{kg}$, i.p.) and implanted with 23 -gauge guide cannulas $(15 \mathrm{~mm}$ hypodermic stainless steel; A-M Systems) targeting either the dorsomedial PFC (dmPFC; $+3.0 \mathrm{~mm} \mathrm{AP,} \pm 0.6 \mathrm{~mm} \mathrm{ML},-4.2 \mathrm{~mm} \mathrm{DV}$ ), ventromedial PFC (vmPFC: $+4.0 \mathrm{~mm} \mathrm{AP}, \pm 0.6 \mathrm{~mm} \mathrm{ML},-5.0 \mathrm{~mm}$ $\mathrm{DV}), \mathrm{OFC}(+3.7 \mathrm{~mm} \mathrm{AP}, \pm 2.6 \mathrm{~mm} \mathrm{ML},-4.7 \mathrm{~mm} \mathrm{DV})$, or anterior insular cortex (AIC; $+1.2 \mathrm{~mm} \mathrm{AP,} \pm 5.5 \mathrm{~mm} \mathrm{ML},-5.7 \mathrm{~mm} \mathrm{DV}$ ) (Paxinos and Watson, 1998). All coordinates were taken with respect to bregma. Cannulas were fixed with dental acrylic cement and anchored with two surgical screws placed on the skull. Stainless steel obturators were inserted into the guide cannulas to prevent clogging until infusions were made. The tips of the cannulas were aimed 0.8 $\mathrm{mm}$ above the target structure. After surgery, animals received food and water ad libitum for $7 \mathrm{~d}$ to allow full recovery before experiments.

\section{Behavior}

All behavioral tasks were performed in home cages through all phases of the experiments. Four types of behavioral taste-guided tasks were used. One task involved behavior guided by innate taste stimuli and three tasks involved behavior guided by learned taste stimuli. All CTA experiments required that rats acquire aversion to an unfamiliar taste (saccharin solution $0.1 \%$ ) when followed ( $15 \mathrm{~min}$ ) by induction of digestive malaise using lithium chloride ( $\mathrm{LiCl} ; 0.4 \mathrm{M} ; 7.5 \mathrm{mg} / \mathrm{kg}$, i.p.). To test CTA memory, animals were presented with the conditioned stimulus (CS; saccharin) without the unconditioned stimulus (US; $\mathrm{LiCl}$ ). Behavioral sessions consisted of taste-sampling tests and a thirst test using a single extra bottle. The taste-sampling tests consisted on the presentation of $12 \mathrm{ml}$ of liquid solution for $10 \mathrm{~min}$ (in one or four bottles). This was followed by a thirst test that evaluated the motivation to drink independently of the test-sampling session and consisted of the presentation of a single bottle with $6 \mathrm{ml}$ of water for $10 \mathrm{~min}$. On each session (taste-sampling test and thirst test), rats were allowed to consume a total of $18 \mathrm{ml}$ of liquid solution. All bottles were weighed before and after each session to measure the total amount of liquid intake. Three different tasks that involved CTA were developed.

No-choice CTA task. This task involves the use of a single bottle throughout the different experimental stages (baseline water consumption sessions and taste-sampling tests). This is a standard CTA task that does not involve choice between options. In no-choice CTA learning, animals were presented with a single bottle containing $6 \mathrm{ml}$ of water, followed by $12 \mathrm{ml}$ of saccharin solution as the CS and LiCl injection as the US. This was important to ensure that animals associate the last taste (saccharin) with the US. In the no-choice CTA memory test, animals were presented with a single bottle containing $12 \mathrm{ml}$ of saccharin solution for 10 minutes, followed by $6 \mathrm{ml}$ of a water thirst test.

Choice CTA task. This task involves the use of four bottles presented simultaneously throughout the experiment. This is a modified version of the one-bottle standard CTA task. This CTA task involves an explicit choice between alternative options presented simultaneously. In choice CTA learning, animals were presented with 4 bottles: 2 bottles, $3 \mathrm{ml}$ each, contained the unfamiliar saccharin solution and 2 bottles, $3 \mathrm{ml}$ each, contained the familiar water solution (total $12 \mathrm{ml}$ of liquid intake: $6 \mathrm{ml}$ of saccharin and $6 \mathrm{ml}$ of water). This was followed by a $6 \mathrm{ml}$ bottle of saccharin (instead of a water thirst test) to make sure the last taste was paired with the $\mathrm{LiCl}$ injection. In the choice CTA memory test, animals were presented with 4 bottles: 2 bottles, $3 \mathrm{ml}$ each, contained the conditioned saccharin solution and 2 bottles, $3 \mathrm{ml}$ each, contained the safe water solution (total $12 \mathrm{ml}$ of liquid intake: $6 \mathrm{ml}$ of saccharin and $6 \mathrm{ml}$ of water). This was followed by a thirst test using $6 \mathrm{ml}$ of water. Bottles of saccharin and water were alternated and changed their position in every session to prevent rats from learning the location of the tastes. We used four bottles rather than two because, with four bottles, we increase the choice options (increased cognitive demand) while decreasing the bias to 
choose by chance for each bottle (from $50 \%$ to $25 \%$ ). To avoid stimulus discrimination based on visual appearance of the reinforcer (bottle and solution), all bottles had the same physical appearance (transparent polycarbonate tubes) and all solutions had the same visual appearance (clear solution)

Complex no-choice CTA task. Similar to the choice CTA task, this task involves the use of four bottles presented simultaneously for all parts of the experiment. However, similar to the no-choice CTA task, this task does not involve choice behavior because animals are presented with all 4 bottles, each containing $3 \mathrm{ml}$ of the same saccharin solution. This was followed by a $6 \mathrm{ml}$ water thirst test. By presenting not one but four bottles at a time with the same taste stimulus, this task represents a standard CTA (without choice involved) with increased cognitive demands (four rather than one bottle).

Innate choice task. Similar to the choice CTA task, this task involves the use of four bottles presented simultaneously throughout experiment. However, in this task, there was no learning involved. Animals were presented with 2 bottles, $3 \mathrm{ml}$ each, of a naturally preferred sweet taste solution (saccharin solution $0.1 \%$ ) and 2 bottles, $3 \mathrm{ml}$ each, of a naturally aversive bitter taste solution (quinine solution $0.02 \%$ ).

\section{Drug infusions}

The day before testing, rats were habituated for infusion handling and injectors were briefly inserted into the cannulas without infusing. Injector tips extended $0.8 \mathrm{~mm}$ beyond the guide cannula. Muscimol and baclofen (M\&B; Sigma-Aldrich) or muscimol alone (MUS) were used to enhance $\mathrm{GABA}_{\mathrm{A}}$ and $\mathrm{GABA}_{\mathrm{B}}$ receptor activity, thereby inactivating the targeted brain region. The inactivating drug was prepared on the day of the infusion using a physiologically filtered saline solution (SAL) as vehicle and infused $10 \mathrm{~min}$ before behavioral testing. M\&B (0.125 ng of each drug/0.5 $\mu \mathrm{l}$ per side) or SAL was infused at a rate of $0.4 \mu \mathrm{l} / \mathrm{min}$ into $\mathrm{dmPFC}, \mathrm{vmPFC}$, and OFC and MUS or SAL was infused at a rate of 10 $\mu \mathrm{l} / \mathrm{min}$ into AIC (5 $\mu \mathrm{g} / 1.0 \mu \mathrm{l}$ per side). Cannulas were connected via polyethylene tubing to $10 \mu$ l syringes (Hamilton) driven by a programmable microinfusion pump (KD Scientific). Dosages and volumes of GABAergic agonists were based on pilot experiments in our laboratory and previous studies targeting the same brain structures (Berman et al., 2000; Bravo-Rivera et al., 2014) where dosages did not affect general locomotion. After infusions, injectors were left in place for $2 \mathrm{~min}$ to allow the drug to diffuse.

\section{Histology}

After behavioral experiments, rats were deeply anesthetized with chloral hydrate $(400 \mathrm{mg} / \mathrm{kg}$, i.p. $)$ and transcardially perfused with $0.9 \%$ saline solution. Brains were then removed and stored in a $10 \%$ formalin solution (Sigma-Aldrich) for at least $3 \mathrm{~d}$. Then, formalin was replaced by a $30 \%$ sucrose solution until tissue saturation was reached. Using a cryostat (Leica, CM1520), brains were cut in $40-\mu \mathrm{m}$-thick coronal sections and mounted on slides. To determine the cannula's location, mounted slides with coronal sections were stained for Nissl bodies and examined under a bright-field microscope (Nikon, H550S). Only rats with cannula locations within the borders of each of the target structures were included in the statistical analysis.

\section{Immunohistochemistry}

Ninety minutes after the end of the last behavioral test, rats were anesthetized with chloral hydrate $(400 \mathrm{mg} / \mathrm{kg}$, i.p. $)$ and then transcardially perfused with $0.9 \%$ saline solution followed by $200 \mathrm{ml}$ of cold $4 \%$ paraformaldehyde (PFA) in $0.1 \mathrm{M}$ phosphate buffer saline (PBS). Brains were removed and fixed overnight in 4\% PFA and then transferred from $20 \%$ sucrose to $30 \%$ sucrose in $0.1 \mathrm{~m}$ PBS for $48 \mathrm{~h}$ for cryoprotection. Frozen sections were cut coronally $(40 \mu \mathrm{m})$ with a cryostat (Leica, CM1520) at different levels of the orbitofrontal and insular cortices.

All sections were washed in $0.1 \mathrm{~m}$ TBS with $0.1 \%$ Tween-20 (SigmaAldrich) between reactions 6 times for $10 \mathrm{~min}$ each. Sections were initially blocked in a solution of $4 \%$ normal goat serum (Vector Laboratories), $4 \%$ bovine serum albumin (Santa Cruz Biotechnology), and $0.1 \%$ Triton X-100 in 0.1 м TBS with $0.1 \%$ Tween, $\mathrm{pH} 7.4$, for $2 \mathrm{~h}$. Afterward, sections were incubated $48 \mathrm{~h}$ at room temperature with antic-Fos serum raised in rabbit polyclonal antibody (Santa Cruz Biotech- nology, sc-52 RRID: AB_2106783; lot A1107) at a concentration of 1:250. Sections were then incubated for $1 \mathrm{~h}$ at room temperature in a solution of biotinylated goat anti-rabbit IgG (Vector Laboratories, BA-1000 RRID: AB_2313606; lot 117429) at a concentration of 1:250 and placed in a mixed avidin biotin horseradish peroxidase complex solution (ABC Elite Kit; Vector Laboratories) for $1 \mathrm{~h}$. Black/brown nuclei immunolabeled for c-Fos were visualized after 10 minutes of exposure using the peroxidase substrate kit (SK-4100, DAB, Vector Laboratories). The reaction was stopped using three $5 \mathrm{~min}$ washes of $0.1 \mathrm{M}$ PBS without Tween. Sections were mounted on gelatin-coated slides and coverslipped.

c-Fos-immunolabeled neurons were counted automatically using ImageJ software (RRID: SCR_003070) at $10 \times$ magnification with a brightfield microscope (Nikon, H550S) equipped with a digital camera. Micrographs were generated at different anterio-posterior (AP) levels from bregma for AIC $(+1.20$ to $+1.50 \mathrm{AP})$ and OFC $(+3.00$ to +3.70 AP). Examples of micrographs are shown in Figure 4. Neurons were considered positive for c-Fos-like immunoreactivity if the nucleus was distinct from the background and was of the appropriate size (area from 25 to $250 \mu^{2}$ ) and shape (at least $60 \%$ circularity). The c-Fosimmunolabeled neuron counts were averaged for each hemisphere in two to three different sections for each structure. The density of c-Fospositive neurons (neurons per square millimeter) was calculated by dividing the number of c-Fos-positive neurons by the total area of each region.

\section{Data collection and analysis}

Baseline water consumption intake was calculated twice a day for the length of the experiment with either one or four bottles. The amount of taste stimulus (saccharin and/or quinine) consumed is expressed as percentage of the baseline water consumption intake (percentage of taste stimulus/water) calculated as follows:

$$
\frac{\text { Saccharin or quinine consumption }}{\text { Mean baseline water consumption }} * 100
$$

A value of 0 indicates complete aversion to the taste stimulus and a value of 100 indicates taste preference (as much as water). When rats were presented with four bottles at the same time, the baseline water consumption and the taste stimulus intake (saccharin and/or quinine solution) was obtained as the sum of the intakes of the corresponding two bottles. Experimental groups were compared by using, when appropriate, paired or unpaired Student's two-tailed $t$ tests or repeated-measures ANOVA followed by post hoc Tukey's honest significant difference test (STATISTICA; StatSoft; RRID:SCR_014213).

\section{Results}

Inactivation of dorsal or ventral mPFC does not affect retrieval of choice CTA

To evaluate the role of $\mathrm{mPFC}$ in choice behavior guided by learned taste aversion, we compared the retrieval of choice CTA before and after pharmacological inactivation of the two main subdivisions of the mPFC (dorsal and ventral). Early on day 1, rats were conditioned to avoid a novel taste stimulus ( saccharin) by pairing it with induced digestive malaise. Six hours later, rats were tested for taste aversion memory to saccharin (test 1 ). We used this first memory retrieval test to match groups (experimental and control) based on similar taste aversion levels. This matching allows for a fair comparison of taste aversion levels before and after local brain inactivations.

The next day (day 2), to evaluate the effect of local mPFC inactivation, we infused either SAL or a GABAergic agonist mixture $(M \& B)$ before a second retrieval test (test 2$)$. In separate groups of animals, we inactivated the dmPFC and vmPFC. The dmPFC includes prelimbic and anterior cingulate cortices, whereas the vmPFC includes infralimbic and medial orbitofrontal cortices. We found that inactivation of dmPFC did not affect retrieval of choice CTA (Fig. 1A), as indicated by the similar levels of saccharin solution consumed with respect to baseline water 


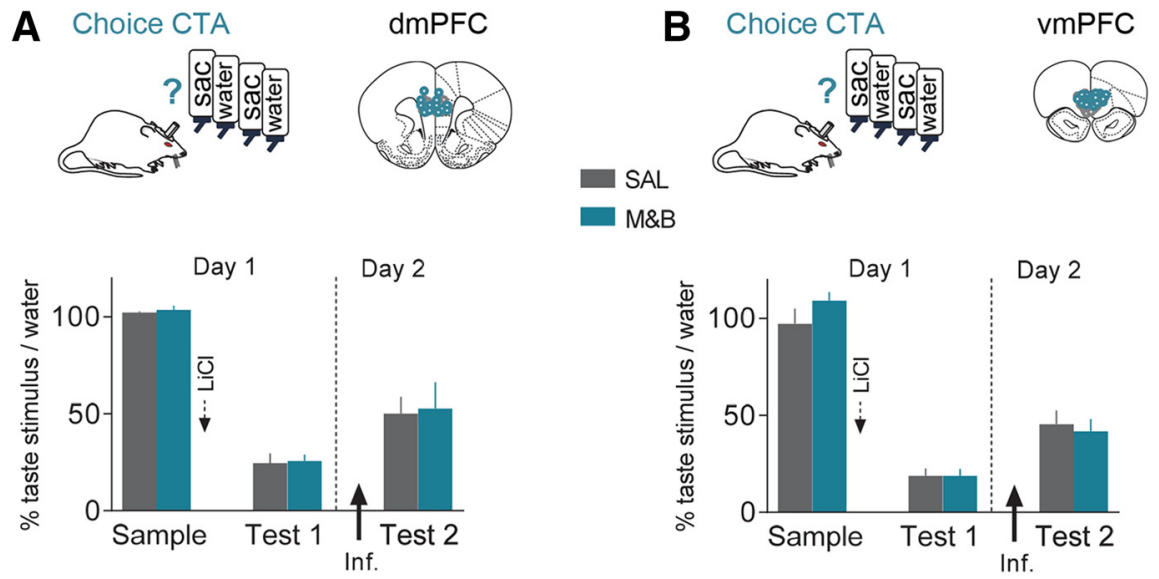

Figure 1. Inactivation of mPFC did not affect retrieval of choice behavior guided by learned taste aversion. $\boldsymbol{A}$, Top left, Schematic representation of the four-bottle CTA task (choice (TA). Top right, Coronal drawings of rat brain atlas showing the placements of the injector tips in dmPFC. Bottom, Infusion of muscimol and baclofen into dmPFC before test 2 did not affect retrieval of choice (TA (M\&B, $n=7 ;$ SAL, $n=7$ ). $\boldsymbol{B}$, Top left, Schematic representation of the four-bottle (TA task (choice CTA). Top right, Coronal drawings of rat brain atlas showing the placements of the injector tips in vmPFC. Bottom, Infusion of muscimol and baclofen into vmPFC before test 2 did not affect retrieval of choice $C T A(M \& B, n=11 ; S A L, n=11)$. Solid arrows indicate time of infusion. Dotted arrows indicate injection of LiCl. Error bars indicate SEM. Sac, Saccharin solution.

consumption (percentage of taste stimulus/water) during test 2 across groups (M\&B: $52.8 \%$; SAL: $49.1 \%$; group effect: $F_{(1,12)}=$ $0.07, p=0.8)$. Similarly, we found that inactivation of vmPFC did not affect the retrieval of choice CTA (Fig. $1 B$ ), as indicated by the similar levels of saccharin solution consumed with respect to baseline water consumption during test 2 across groups (M\&B: 41.8\%; SAL: $45.56 \%$; group effect: $\left.F_{(1,20)}=0.2, p=0.6\right)$. We also found that inactivation of dmPFC (M\&B: 101.2\%; SAL: $123.9 \%$; $\left.t_{(12)}=1.08, p=0.3\right)$ or vmPFC (M\&B: $124.3 \%$; SAL: $127.2 \%$; $t_{(20)}$ unpaired $\left.=-0.14, p=0.8\right)$ did not affect motivation to drink water, as indicated by similar levels of water consumed in the extra bottle between groups $(p>0.05$, data not shown; from here on, extra bottle data appear in text only when percentage taste stimulus/water differed between groups after infusion). Therefore, these results suggest that $\mathrm{mPFC}$ (including dmPFC and vmPFC) is not necessary for the retrieval of choice behavior guided by taste aversion.

\section{Inactivation of OFC blocks retrieval of choice CTA}

We next investigated another prefrontal region known to be involved in taste processing, emotional processing, and action selection: the OFC (for review, see Wallis, 2012; Stalnaker et al., 2015). After matching for similar levels of CTA on day 1 , rats were infused with either SAL or M\&B into the OFC on day 2 (Fig. 2A). We found that SAL-infused rats showed low levels of saccharin consumption similar to test 1 , indicating learned aversion to saccharin. However, OFC-inactivated rats showed significantly higher levels of saccharin consumption during test 2 (M\&B: 72.5\%; SAL: $27.6 \%$; group effect: $F_{(1,14)}=5.5, p=0.03$; trial effect: $F_{(2,28)}=91.5, p<0.001$; interaction effect: $F_{(2,28)}=10.1$, $p=0.0003$; post hoc comparison between M\&B and SAL in test 2: $p=0.0002)$, similar to preconditioning, drug-free levels (M\&B before conditioning: $91.5 \%$ vs $M \& B$ at test $2: 72.5 \%$; $t_{(8)}$ paired $=$ $1.6, p=0.1)$, suggesting a full blockade of choice CTA retrieval. Importantly, the OFC inactivation effect was not due to lack of motivation to drink because inactivated- and saline-treated rats consumed similar amounts of water from the extra bottle (M\&B: 118.6\%; SAL: $107.7 \%$; $t_{(14)}$ unpaired $\left.=-0.5, p=0.6\right)$. The effect of OFC inactivation on the retrieval of choice CTA can be inter- preted as evidence for a role of OFC in the retrieval of CTA memory or a specific role of OFC in choice behavior guided by learned taste aversion.

\section{Inactivation of OFC does not affect retrieval of no-choice CTA, even with increased task difficulty}

Because our choice CTA task is based on the retrieval of CTA memory and because OFC contains the secondary taste cortex (Rolls, 2004; Price, 2007), we tested whether OFC inactivation impairs the retrieval of a CTA task that does not involve choice behavior (no-choice CTA; Fig. 2B). We found that inactivation of OFC before memory test did not affect the retrieval of CTA, as indicated by similar levels of saccharin solution consumed with respect to baseline water consumption during test 2 between groups (M\&B: 47.3\%; SAL: $42.5 \%$; group effect: $F_{(1,15)}=0.02, p=$ $0.9)$. This result corroborates that indeed OFC guides choice behavior rather than mediating retrieval of CTA memory and is consistent with previous reports showing that OFC lesions do not impair CTA (Gallagher et al., 1999). Therefore, these findings rule out that the observed OFC inactivation effect on retrieval of choice CTA was simply due to an impairment to retrieve the CTA memory, which in turn indicates that OFC does not appear to be a storage site for taste aversion memories.

Choice-related activity in prefrontal regions can be confounded with task difficulty. The use of four bottles in the choice CTA compared with one bottle in the no-choice CTA represents an increase in cognitive demand. CTA tasks using several bottles with two different tastes likely require maintaining the information of each taste stimulus in working memory for comparison and OFC activity has been correlated with such cognitive demand in monkeys (Lara et al., 2009). To assess whether increasing the level of complexity between tasks (one bottle no-choice tasks vs four bottle choice tasks) could explain the observed OFC inactivation impairment on choice CTA, we inactivated OFC before the retrieval of a CTA task that involved the presentation of four bottles (all bottles containing saccharin), but that does not involve a choice (complex no-choice CTA; Fig. 2D). We found that inactivation of OFC did not affect the retrieval of complex nochoice CTA, as indicated by the similar levels of saccharin solution consumed with respect to baseline water consumption during test 2 across groups (M\&B: 39.6\%; SAL: 37.9\%; group effect: $\left.F_{(1,18)}=0.2, p=0.6\right)$. Therefore, these results rule out two possibilities: (1) that the OFC inactivation effect on choice CTA retrieval is simply due to increased cognitive demands of the task and (2) that the OFC inactivation effect on choice CTA retrieval is due to inability to compare several taste sampling solutions at a time. Together, these results support the notion that OFC plays a particular key role in choice guided by learned taste aversion.

Inactivation of OFC does not affect acquisition of choice CTA If OFC is necessary to retrieve a CTA memory when choice is involved, it is also possible that OFC is required for learning the initial contingencies that lead to the formation of choice CTA memory. In addition, it is also possible that OFC represents the 
sensory information of the taste stimulus (Rolls, 2004), which is required for taste detection in our task or to choose between any two taste stimuli (saccharin and water) presented simultaneously before learning about their motivational value. To test these possibilities, we inactivated OFC before the initial taste sampling that represents the acquisition phase of choice CTA on day 1 (Fig. 2C). We found that inactivation of OFC did not affect the acquisition of taste aversion learning, as indicated by the similar levels of saccharin solution consumed with respect to baseline water consumption during both retrieval tests on day 1 (M\&B: $15.8 \%$; SAL: $12.7 \%)$ and day 2 across groups $(M \& B$ : $37.7 \%$; SAL: $36.8 \%$; group effect: $F_{(1,10)}=$ $0.3, p=0.6)$. This is consistent with a lack of effect in CTA acquisition after OFC lesion (Gallagher et al., 1999). In addition, we found that OFC inactivation did not affect taste stimuli detection, as indicated by similar levels of saccharin solution consumed with respect to water consumption during taste sampling before the injection of the US LiCl on day 1 compared with the control group (M\&B: 95.2\%; SAL: 95.0\%; $t_{(10)}$ unpaired $\left.=-0.9, p=0.4\right)$. Therefore, these results rule out the possibility that the OFC inactivation effect on choice CTA retrieval is due to a nonspecific impairment in the perception or related behavior to choosing among taste stimuli presented simultaneously.

\section{Inactivation of OFC does not affect choice of innate taste stimuli}

Not all preferences or aversions to taste stimuli are learned. There are taste stimuli that are naturally preferred or aversive; that is, there are taste stimuli that possess innate (unlearned) value. To determine whether OFC is necessary for choices guided by innate taste preference/aversion, we inactivated OFC before the presentation of a naturally preferred sweet (saccharin) versus an aversive bitter (quinine) taste. For direct comparison with our choice CTA task, our innate choice task involved the presentation of two different taste stimuli twice on day 1 (sample and test 1) and once on day 2 (test 2). Preference for saccharin and aversion to quinine with respect to baseline water consumption remained unaltered throughout the experiment (Fig. 2E). We found that OFC inactivation, compared with the control group, did not affect the natural preference for saccharin (M\&B: 106.2\%; SAL: 112.8\%; group effect: $\left.F_{(1,14)}=0.3, p=0.6\right)$ nor the aversion for quinine (M\&B: $43.2 \%$; SAL: $42.5 \%$; group effect: $\left.F_{(1,14)}=0.2, p=0.7\right)$
A
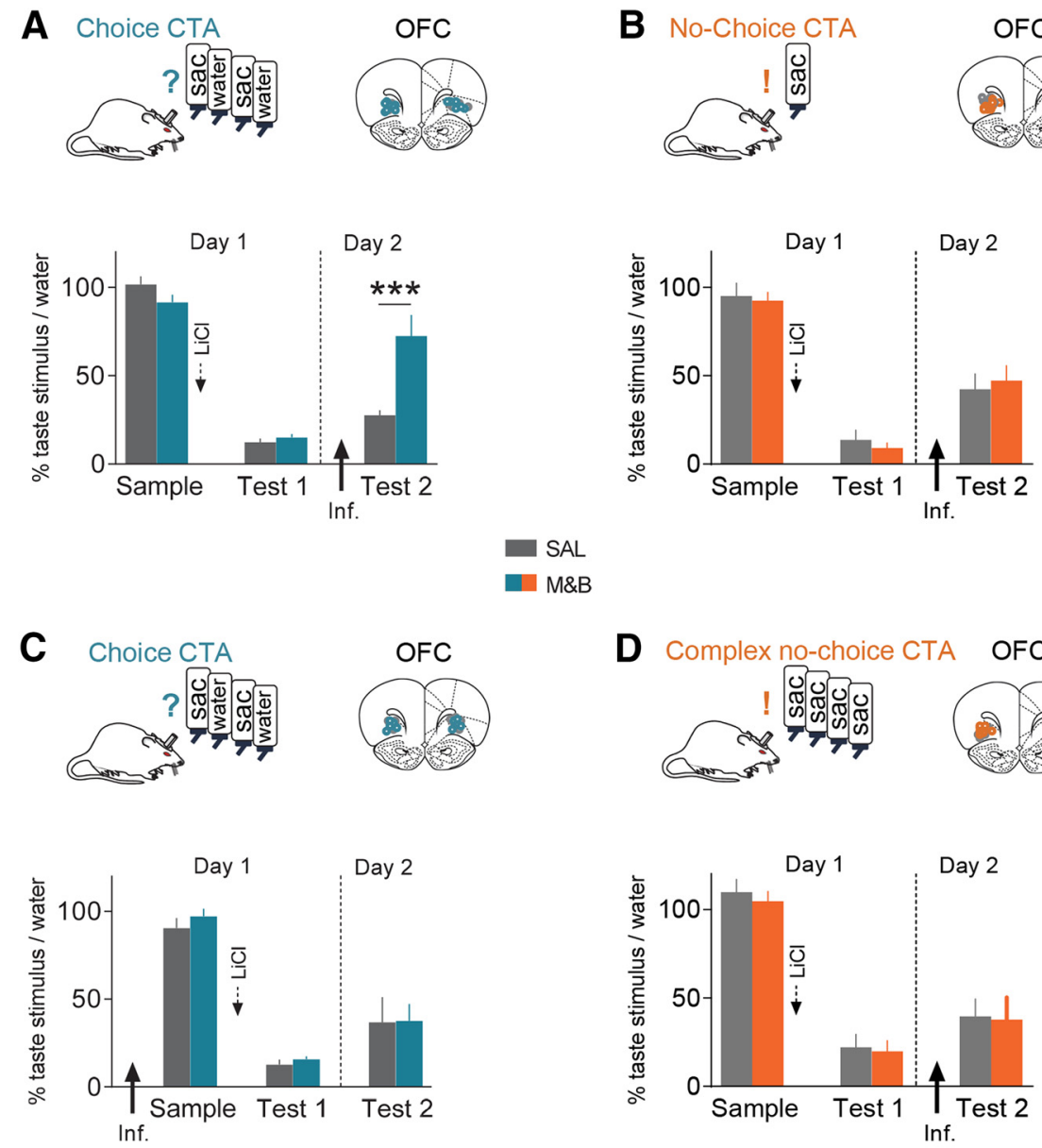

SAL
M\&B
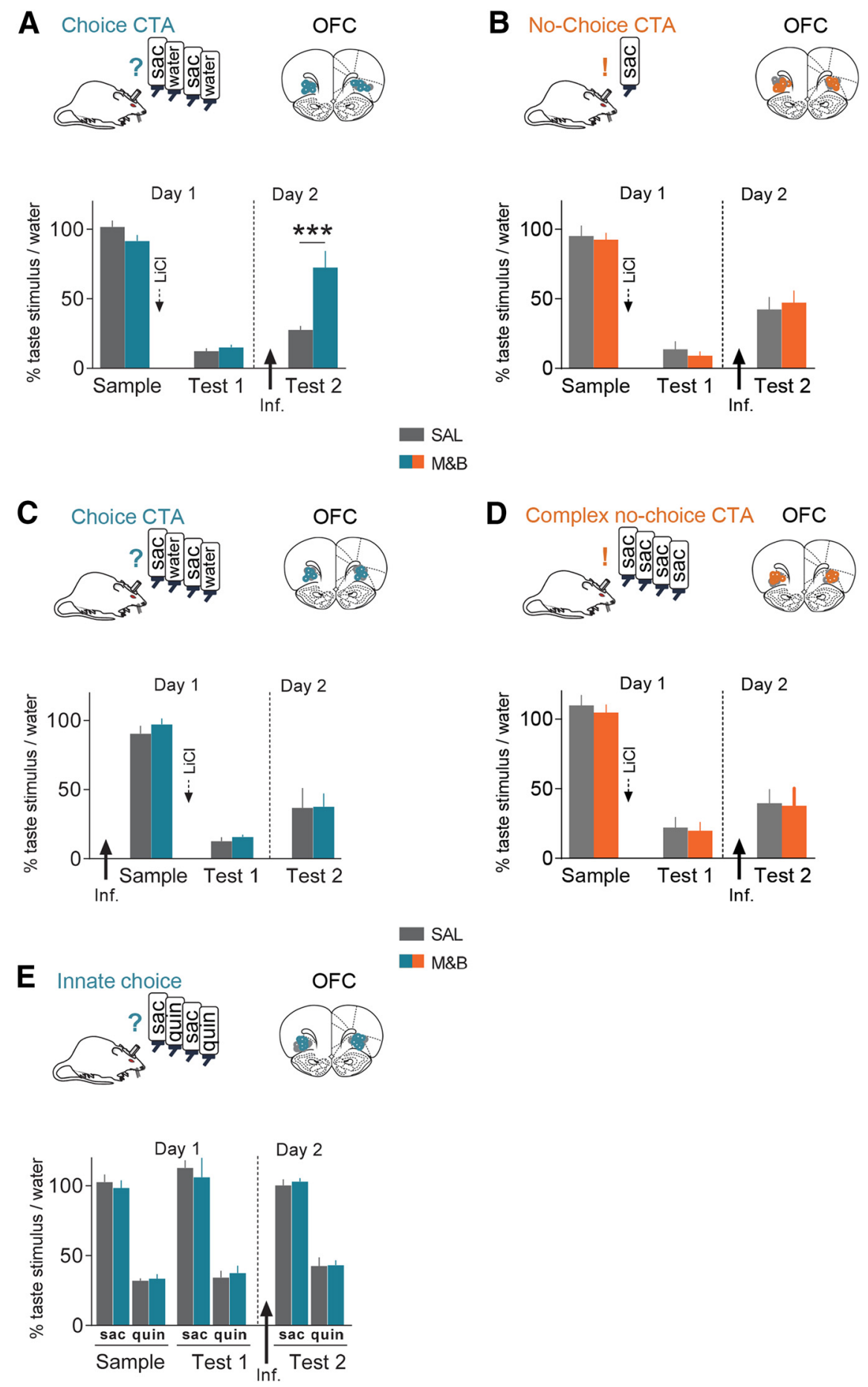

Figure 2. Inactivation of OFC blocked the retrieval of choice behavior guided by learned taste aversion, but left other tasteguided behaviors intact. $A$, Top left, Schematic representation of the four-bottle CTA retrieval task (choice CTA). Lower: Infusion of muscimol and baclofen into OFC before test 2 blocked the retrieval of choice CTA (M\&B, $n=9 ; S A L, n=7$ ). $\boldsymbol{B}$, Top left, Schematic representation of one bottle CTA retrieval task (no-choice (TA). Bottom, Infusion of muscimol and baclofen into OFC before test 2 did not affect retrieval of no-choice CTA (M\&B, $n=9 ; S A L, n=8)$. C, Top left, Schematic representation of the four-bottle CTA retrieval task (choice (TA). Bottom, Infusion of muscimol and baclofen into OFC before taste learning did not affect acquisition or retrieval of choice CTA (M\&B, $n=6 ; S A L, n=6)$. D. Top left, Schematic representation of the four-bottle CTA retrieval task that does not involve options to choose from (complex no-choice (TA). Bottom, Infusion of muscimol and baclofen into OFC before test 2 did not affect the retrieval of complex no-choice (TA (M\&B, $n=7 ; S A L, n=5$ ). $\boldsymbol{E}$, Top left, Schematic representation of the four-bottle nonlearned taste preference task (no-choice (TA). Bottom, Infusion of muscimol and baclofen into $0 \mathrm{FC}$ before test 2 did not affect innate choice behavior ( $M \& B, n=8 ; S A L, n=8$ ). Atlas figures indicate placements of injectors' tips in OFC. Solid arrows indicate time of infusion. Dotted arrows indicate injection of LiCl. In this and subsequent figures, color code is the following: blue represents tasks that involve choice (?) and orange represents tasks that do not involve choice (!). Error bars indicate SEM. Sac, Saccharin solution; Quin, quinine. ${ }^{* *} p<0.001$. 

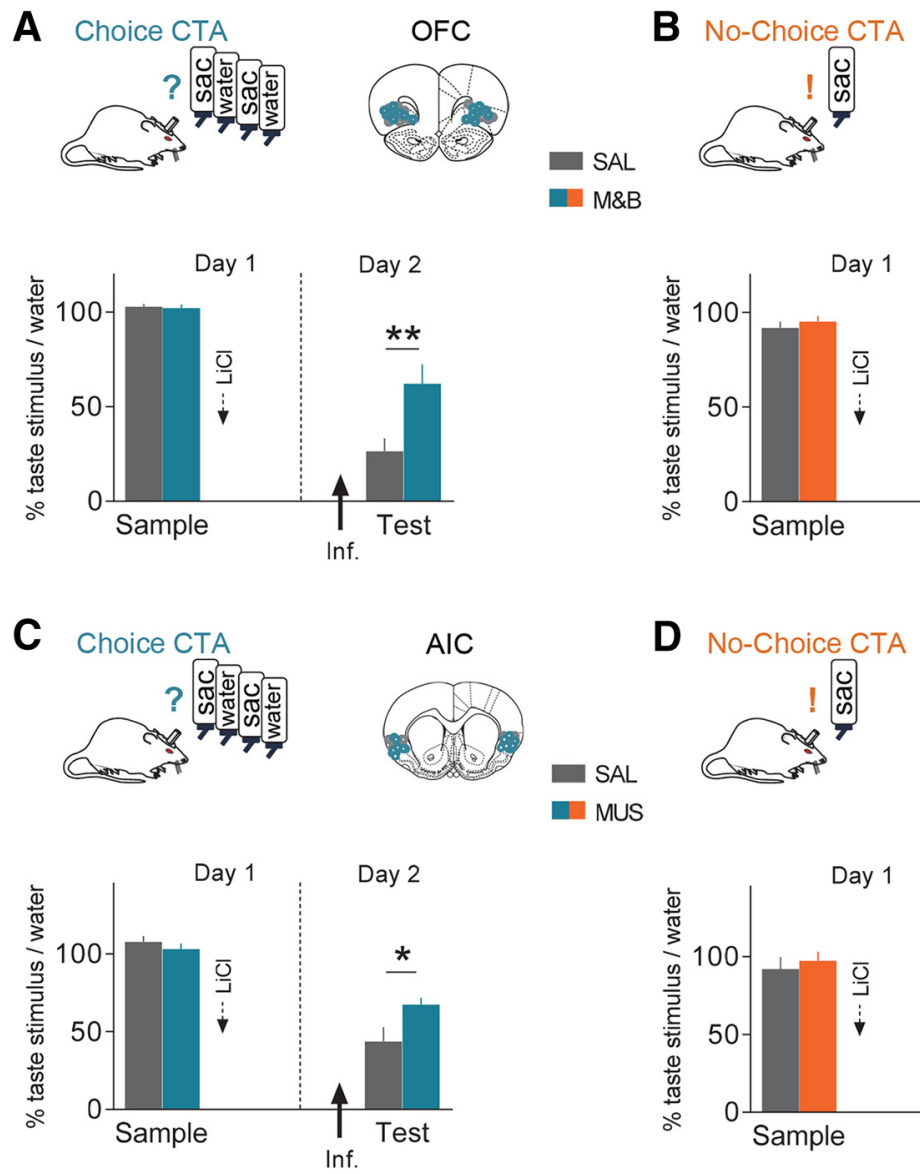

Figure 3. Differential effect of OFC and AIC inactivation on choice CTA versus no-choice CTA. $\boldsymbol{A}$, Top left, Schematic representation of the four-bottle CTA task (choice (TA). Bottom, Infusion of muscimol and baclofen into OFC before test blocked the retrieval of choice CTA (M\&B, $n=8 ;$ SAL, $n=8$ ). $B$, Top left, Schematic representation of one bottle CTA task (no-choice (TA). Bottom, Infusion of muscimol and baclofen into OFC before the test did not affect retrieval of no-choice (TA (M\&B, $n=10 ; S A L, n=6)$. Atlas figures indicate placements of injectors' tips in OFC. C, Top left, Schematic representation of the four-bottle CTA task (choice (TA). Bottom, Infusion of muscimol into AIC before the test impaired the retrieval of choice CTA (MUS, $n=10 ; S A L n=12$ ). $\boldsymbol{D}$, Top left, Schematic representation of one bottle CTA task (no-choice (TA). Bottom, Infusion of muscimol into AIC before test impaired the retrieval of no-choice (TA (MUS, $n=10 ;$ SAL, $n=10$ ). Atlas figures indicate placements of injectors' tips in AIC. Solid arrows indicate time of infusion. Dotted arrows indicate injection of LiCl. Error bars indicate SEM. Sac, Saccharin solution. ${ }^{*} p<0.05$; ${ }^{* *} p<0.01$.

during test 2 on day 2 (post hoc comparisons, all ps $>0.05$ ). Therefore, our results indicate that OFC is not necessary for choices guided by innate taste preference/aversion. These results highlight that OFC contribution to choice behavior involves the use of taste stimuli with incentive value only if it has been acquired through learning.

\section{Inactivation of OFC blocks choice CTA regardless of when retrieval occurs}

At which time point the memory retrieval test is performed may trigger different and dissociable brain mechanisms. To rule out that the OFC inactivation effect that we observed during choice CTA depends on when retrieval test occurs, we inactivated OFC before a first and only retrieval test on day 2. Having two retrieval tests, as in the above experiments, allowed us to ascertain, via group matching, that both experimental and control animals had similar (nonsignificant) retrieval levels to compare before and after brain inactivation. Here, even without a group matching advantage, we replicated the main finding. We found, consistent with OFC inactivation before test 2 (Fig. $2 A$ ), that OFC inactivation before the only test (Fig. $3 A$ ) impaired the retrieval of choice CTA memory compared with the control group (M\&B: $62.2 \%$;

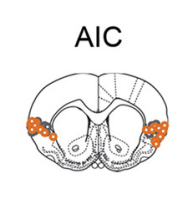

SAL: $26.6 \%$; group effect: $F_{(1,14)}=6.8$, $p=0.02$; trial effect: $F_{(1,14)}=100.7, p<$ 0.001 ; interaction effect: $F_{(1,14)}=9.8, p=$ 0.0003 ; post hoc comparison between $\mathrm{M} \& \mathrm{~B}$ and SAL in test: $p=0.007)$. Similar to OFC inactivation before test 2 , the effect observed during the OFC inactivation before a single test was not due to lack of motivation to drink because inactivatedand saline-treated rats consumed similar amounts of water from the extra bottle (M\&B: $107.2 \%$; SAL: $136.1 \%$; $t_{(14)}$ unpaired $=1.3, p=0.2$ ). Furthermore, consistent with OFC inactivation before test 2 , we found that inactivation of OFC before a single memory test on day 2 did not affect the retrieval of no-choice CTA, as indicated by the similar levels across groups of saccharin solution consumed with respect to baseline water consumption during test (M\&B: 29.7\%; SAL: $35.6 \%$; group effect: $F_{(1,14)}=0.03, p=$ 0.9 ; Fig. $3 B$ ). Therefore, these results suggest that OFC is necessary for choice behavior independently of when the learned taste aversion is retrieved and further support the notion that OFC is not the locus for the CTA memory.

Inactivation of AIC blocks the retrieval of both no-choice and choice CTA

To test the possibility that AIC (primary taste cortex) stores the CTA memory independently of whether it is during a choice or no-choice situation, we inactivated AIC before a retrieval test of both choice and no-choice CTA tasks. Consistent with a role for AIC as the storage site of CTA memory (Gallo et al., 1992; Berman et al., 2000), we found that inactivation of AIC impaired the retrieval of CTA, as indicated by the significantly higher levels of saccharin solution consumed with respect to baseline water consumption compared with control group during both tests of choice (Fig. 3C; MUS: 67.4\%; SAL: 41.1\%; group effect: $F_{(1,20)}=2.3058, p=0.1445$; trial effect: $F_{(1,20)}=77.7, p<0.001$; interaction effect: $F_{(1,20)}=6.3, p=0.02$; post hoc comparison between M\&B and SAL in test: $p=0.04$ ) and no-choice CTA (Fig. 3D; MUS: $62.6 \%$; SAL: $31.6 \%$; group effect: $F_{(1,18)}=4.8088, p=$ 0.04; trial effect: $F_{(1,18)}=49.05, p<0.001$; interaction effect: $F_{(1,18)}=3.5, p=0.07$; post hoc comparison between $\mathrm{M} \& \mathrm{~B}$ and SAL in test: $p=0.03$ ) on day 2. These AIC inactivation effects were not due to impairment in motivation to drink, as indicated by similar levels among groups of water intake with the extra bottle presented at the end of our choice (MUS: 73.5\%; SAL: $75.6 \% ; t_{(20)}$ unpaired $\left.=-0.4, p=0.7\right)$ and no-choice (MUS: $96.5 \%$; SAL: $103.1 \% ; t_{(18)}$ unpaired $=0.9, p=0.4$ ) tasks. Therefore, these results suggest that AIC is the storage site for the CTA memory independently of whether the task involves choice behavior. Furthermore, our findings that OFC inactivation blocks choice but not no-choice CTA and that AIC blocks the retrieval of both choice and no-choice CTA suggest a differential involvement of OFC and AIC in taste-guided tasks. 
Choice CTA increases neuronal activity in the lateral portion of $\mathrm{OFC}$

Our inactivation results suggest that, when taste options are available, OFC appears to be recruited more heavily than when no choice options are available. To test this recruitment issue directly, separate groups of rats underwent choice CTA and no-choice CTA on day 1 and 90 minutes after CTA retrieval test on day 2, all animals were killed and perfused. Both groups showed similar levels of taste aversion after retrieval, as indicated by the similar levels across groups of saccharin solution consumed with respect to baseline water consumption during test 2 (choice CTA: 22.46\%; no-choice CTA: $16.47 \% ; t_{(7)}$ unpaired $\left.=-0.6, p=0.6\right)$. Brains were removed, sectioned, and processed for immunohistochemistry to assess neural activation using the neural activity marker c-Fos. We found increased c-Fos expression in the lateral OFC (choice CTA: 377.7 counts; no-choice CTA: 245.0 counts; $t_{(7)}$ unpaired $=-2.6$, $p=0.04)$, but not in the ventral OFC (choice CTA: 494.1 counts; no-choice CTA: 390.1 counts; $t_{(7)}$ unpaired $=-1.1$, $p=0.3)$, of rats that underwent choice

CTA compared with those that underwent no-choice CTA (Fig. $4 A$ ). Therefore, consistent with our OFC inactivation results, the neural activity marker findings indicate that OFC is recruited when choice behavior is involved. In contrast, and also consistent with our AIC inactivation results, the neural activity marker findings show that AIC does not show differential involvement under the two retrieval conditions, as indicated by the same c-Fos expression levels after choice and no-choice CTA (choice CTA: 291.4 counts; no-choice CTA: 251.4 counts, $t_{(7)}$ unpaired $=$ $-1.5, p=0.2$ ) (Fig. $4 B$ ). Together, these findings indicate that choice CTA recruits the lateral region of the OFC, whereas CTA retrieval, regardless of task conditions (choice or no-choice), similarly recruits the AIC.

\section{Discussion}

We developed a simple learned taste aversion task that allows comparison of the influence of past aversive experiences on behaviors that involve choice versus those behaviors that do not. In a series of experiments using local pharmacological inactivation, we tested four cortical regions, the OFC, AIC, dmPFC, and vmPFC, which were previously associated with taste processing, emotional processing, and decision making, to determine whether they are critical for choice behavior guided by biologically significant taste stimuli. Collectively, our findings indicate that OFC, but not AIC, dmPFC, or vmPFC, is necessary for choice behavior guided by learned, but not innate, taste aversion. Notably, this study provides novel evidence for the recruitment of OFC in guiding choice behavior dissociable from AIC-dependent taste aversion memory (Fig. 5).

\section{OFC is necessary for choice behavior guided by taste aversion memory}

We found that OFC inactivation impaired the ability to select adaptive behavior (approach the water bottle and avoid the saccharin bottle) guided by a previously acquired aversive value of
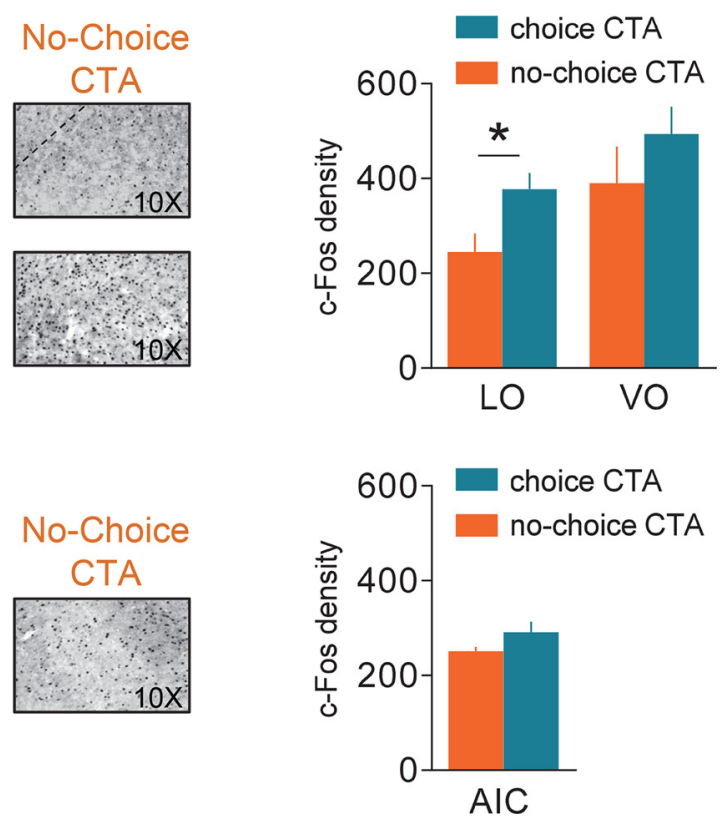

Figure 4. Choice behavior in taste aversion retrieval increases c-Fos expression in lateral OFC, but not in AIC. Comparison of c-Fos and no-choice CTA. $\boldsymbol{B}$, Left, Representative micrographs of c-Fos expression in the AIC at $10 \times$. Right, Group data showing similar c-Fos density in AIC after retrieval of choice CTA and no-choice CTA. Error bars indicate SEM. * $p<0.05$.

the taste stimulus in the choice CTA task regardless of when the taste memory was retrieved. This is consistent with OFC's role in decision making based on the value of the stimulus (Ostlund and Balleine, 2007; Balleine et al., 2011; Wallis, 2012). In contrast, we found that OFC inactivation left intact the ability to select the adaptive behavior (avoid the saccharin bottle) guided by a previously acquired aversive value of the taste stimulus in the nochoice CTA task. OFC may play a particularly important role at the time of choice (Rich and Wallis, 2016). Our results extend previous findings, which involve OFC in value updating using reinforcement devaluation (Pickens et al., 2003; Pickens et al., 2005), by showing that OFC inactivation impairs the ability to use the updated value to guide choice behavior. In turn, this suggests that, although not tested directly here, a different type of devaluation method (e.g., satiation) would likely yield the same results. Therefore, our results support the notion that OFC is particularly necessary for action selection guided by updated valuation of stimulus only when choice behavior is involved and unnecessary for simple value-based behavior (Schoenbaum et al., 2011).

During choice in a changing environment, the brain has to compare flexibly the value of different options available at the same time (Sugrue et al., 2005). OFC neural signals of absolute and relative value have been described previously (Tremblay and Schultz, 1999; Padoa-Schioppa and Assad, 2008; Grabenhorst and Rolls, 2009; Schoenbaum et al., 2011). Arguably, appropriate action selection in the no-choice CTA task relies on retrieval of the absolute value (a quantity related to salience) of the only taste stimuli available (not influenced by the values of other stimuli), whereas our choice CTA task may rely more heavily on the updated relative value of saccharin compared with water. Therefore, similar to what has been reported in humans (Elliott et al., 2008) and monkey OFC (Tremblay and Schultz, 1999), our findings suggest that the rat OFC is critical to represent the updated relative, rather than absolute (PadoaSchioppa and Assad, 2008), valuation of the stimuli at the time of 


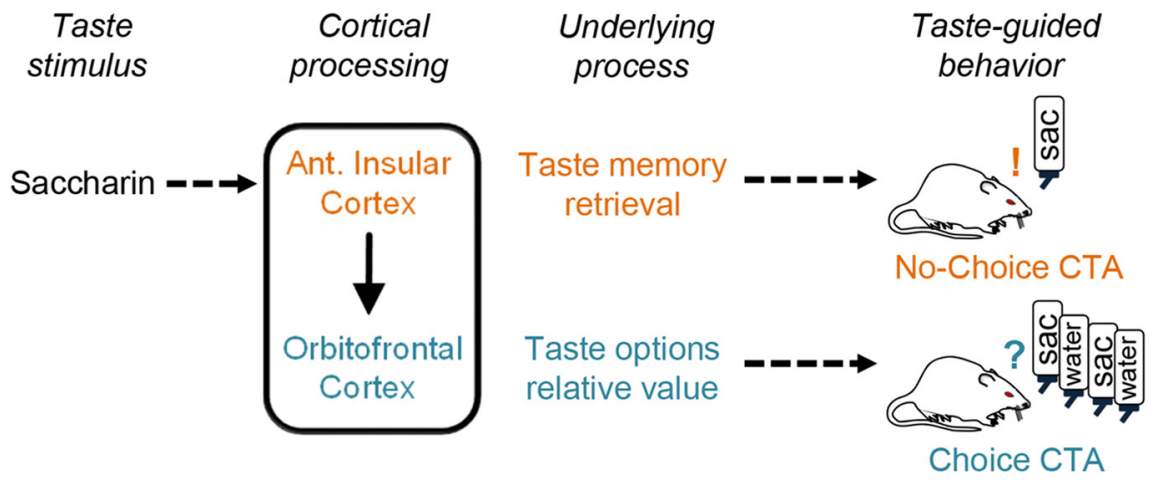

Figure 5. Proposed model for cortical processing in choice behavior guided by the retrieval of taste aversion memory. For the retrieval of a taste-aversive memory without choice (no-choice (TA), activity of the AIC is recruited and may be sufficient. In contrast, for the retrieval of a taste aversion to guide choice behavior (choice (TA), the AIC may be required to retrieve the memory and $\mathrm{OFC}$ recruited to compute the relative value of the options available and to execute the appropriate action to choose.

ever, unlike OFC, we found that neither the dorsal nor ventral mPFC was necessary for choice behavior guided by tasteaversive memories. It is possible that OFC information about choice CTA bypasses the MPFC to engage an interface between limbic values and motor goals such as the ventral striatum directly; experiments are under way to test this possibility.

\section{AIC is necessary for retrieval of taste aversion memories}

OFC contains the secondary taste cortex because it receives strong functional projections from the primary taste cortex (Rolls, 2004; Price, 2007), which is also known as the AIC. AIC has been reported to be involved in behavior guided by goal-

choice. Although speculative, it appears that the deficits we found in OFC-inactivated rats may be due to the loss of the ability to compare relative values during choice.

Because OFC activity has been shown to be involved in many cognitive abilities and its malfunction is implicated in many mental disorders (Rolls, 2004; Wallis, 2012), it is important, not only to identify what the OFC does, but also what it does not do (Stalnaker et al., 2015). It is possible that OFC is not only necessary for choice behavior guided by tastes that acquire value through learning, but also when choosing between taste stimuli with innate value (e.g., naturally sweet vs naturally bitter). Because we found that OFC inactivation did not affect choice behavior when it involved options that possess an innate value (saccharin vs quinine), the relative value computed in OFC is based on learned values. This is consistent with OFC's role in using associative learning to control behavior (Gallagher et al., 1999; Schoenbaum and Roesch, 2005), as well as with the reported lack of taste cortex involvement in unconditioned taste reactivity tests (King et al., 2015). In addition, our results suggest that OFC may be unnecessary to assign relative value to the taste stimuli during acquisition of choice CTA. Rather, OFC appears to be necessary when updating the outcome of a previously assigned value during retrieval. Therefore, similar to OFC-damaged patients and OFC-lesioned nonhuman primates (Wallis, 2012), OFC-inactivated rats in the current study showed many intact cognitive abilities, but were profoundly impaired specifically in flexibly applying learned emotional information about previous stimuli experiences to make a choice.

\section{mPFC is not necessary for choice behavior guided by taste aversion memory}

Compared with primate PFC, less is known about the functional differentiation across several areas of the rodent PFC involved in value-based choice behavior. Few rat studies have compared different regions of PFC under the same experimental conditions involving choice behavior. In addition to OFC, the medial wall of the rat PFC is prominently involved in emotional processing and executive behaviors (Sotres-Bayon and Quirk, 2010). OFC strongly projects to mPFC (Hoover and Vertes, 2011) and has consistently been involved in taste coding (Jezzini et al., 2013) and control of behavior guided by learned taste stimuli (Hernádi et al., 2000; Mickley et al., 2005; Akirav et al., 2006; Lin et al., 2010; Jezzini et al., 2013; Marotta et al., 2014; Xin et al., 2014; Gonzalez et al., 2015). Therefore, mPFC was an obvious candidate prefrontal region to evaluate in the choice CTA task. Unexpectedly, how- directed actions (Parkes and Balleine, 2013) and risk taking (Ishii et al., 2012), but is most widely recognized for its long-standing role in storing taste-aversive memories after CTA (Gallo et al., 1992; Bermúdez-Rattoni et al., 1997; Berman et al., 2000). Consistent with its role in memory and action selection guided by motivational information, we found that AIC inactivation impaired retrieval of taste aversion memory independently of whether this is under choice or no-choice circumstances. These findings are also consistent with the role of AIC in reinforcer devaluation regardless of whether the behavioral test involves choice behavior (Machado and Bachevalier, 2007). Together, these findings suggest that AIC may store emotional information containing the basic features and associative updated value of taste stimuli that is sufficient to select the appropriate response when no other taste options are available (i.e., during no-choice CTA retrieval). When taste options are available, however, OFC appears to recruit AIC activity to retrieve taste memories and to guide choice behavior (i.e., during choice CTA retrieval; Fig. 5).

\section{Choice behavior recruits the lateral region of OFC, but not AIC}

Consistent with our inactivation results, we found that choice behavior in taste aversion retrieval recruits OFC, but not AIC. Notably, within the OFC of the rat, our neuronal activity results indicate that choice behavior recruits a lateral network rather than a medial network. This is consistent with the emerging notion that the more medial versus more lateral regions of OFC code for different correlates of value information (Rich and Wallis, 2014; Stalnaker et al., 2015).

\section{Cortical processing of taste experiences to guide choice behavior}

Despite the interest generated by cortical areas such as the AIC and OFC, due to their involvement in psychiatric disorders characterized by emotional decision-making deficits, their distinct contribution to choice behavior is not understood. Our findings suggest that, when an individual needs to compare a set of previous emotional taste experiences, the AIC relays the stored representation of the absolute value of each taste stimuli to the OFC. Then, OFC may use the representation of the stimuli that acquired incentive value through association with a negative outcome to calculate the relative value by comparing the taste associations available and thereby aid in guiding choice behavior. This interpretation is consistent with the notion that OFC uses the updated learned value of a sensory stimulus as incentive to 
guide choice behavior (Schoenbaum and Setlow, 2001; Ostlund and Balleine, 2007; Balleine et al., 2011; Parkes and Balleine, 2013). Finally, our findings are also consistent with a recent report showing that neuronal activity of both AIC and OFC correlate with distinct aspects of choice behavior guided by taste stimuli (MacDonald et al., 2009). Notably, our study provides novel evidence for the key role of OFC in guiding choice behavior that is dissociable from the adjacent AIC-dependent taste aversion memory.

\section{References}

Akirav I, Khatsrinov V, Vouimba RM, Merhav M, Ferreira G, Rosenblum K, Maroun M (2006) Extinction of conditioned taste aversion depends on functional protein synthesis but not on NMDA receptor activation in the ventromedial prefrontal cortex. Learn Mem 13:254-258. CrossRef Medline

Balleine BW, Leung BK, Ostlund SB (2011) The orbitofrontal cortex, predicted value, and choice. Ann N Y Acad Sci 1239:43-50. CrossRef Medline

Berman DE, Hazvi S, Neduva V, Dudai Y (2000) The role of identified neurotransmitter systems in the response of insular cortex to unfamiliar taste: activation of ERK1-2 and formation of a memory trace. J Neurosci 20: 7017-7023. Medline

Bermúdez-Rattoni F (2004) Molecular mechanisms of taste-recognition memory. Nat Rev Neurosci 5:209-217. CrossRef Medline

Bermúdez-Rattoni F, Introini-Collison I, Coleman-Mesches K, McGaugh JL (1997) Insular cortex and amygdala lesions induced after aversive training impair retention: effects of degree of training. Neurobiol Learn Mem 67:57-63. CrossRef Medline

Bissière S, Plachta N, Hoyer D, McAllister KH, Olpe HR, Grace AA, Cryan JF (2008) The rostral anterior cingulate cortex modulates the efficiency of amygdala-dependent fear learning. Biol Psychiatry 63:821-831. CrossRef Medline

Bravo-Rivera C, Roman-Ortiz C, Brignoni-Perez E, Sotres-Bayon F, Quirk GJ (2014) Neural structures mediating expression and extinction of platformmediated avoidance. J Neurosci 34:9736-9742. CrossRef Medline

Bures J, Bermúdez-Rattoni F, Yamamoto T (1998) Conditioned taste aversion: memory of a special kind. Oxford: OUP.

Corcoran KA, Quirk GJ (2007) Activity in prelimbic cortex is necessary for the expression of learned, but not innate, fears. J Neurosci 27:840-844. CrossRef Medline

Elliott R, Agnew Z, Deakin JF (2008) Medial orbitofrontal cortex codes relative rather than absolute value of financial rewards in humans. Eur J Neurosci 27:2213-2218. CrossRef Medline

Gallagher M, McMahan RW, Schoenbaum G (1999) Orbitofrontal cortex and representation of incentive value in associative learning. J Neurosci 19:6610-6614. Medline

Gallo M, Roldan G, Bures J (1992) Differential involvement of gustatory insular cortex and amygdala in the acquisition and retrieval of conditioned taste aversion in rats. Behav Brain Res 52:91-97. CrossRef Medline

Gonzalez MC, Villar ME, Igaz LM, Viola H, Medina JH (2015) Dorsal medial prefrontal cortex contributes to conditioned taste aversion memory consolidation and retrieval. Neurobiol Learn Mem 126:1-6. CrossRef Medline

Grabenhorst F, Rolls ET (2009) Different representations of relative and absolute subjective value in the human brain. Neuroimage 48:258-268. CrossRef Medline

Hernádi I, Karádi Z, Vígh J, Petykó Z, Egyed R, Berta B, Lénárd L (2000) Alterations of conditioned taste aversion after microiontophoretically applied neurotoxins in the medial prefrontal cortex of the rat. Brain Res Bull 53:751-758. CrossRef Medline

Holmes NM, Marchand AR, Coutureau E (2010) Pavlovian to instrumental transfer: a neurobehavioural perspective. Neurosci Biobehav Rev 34: 1277-1295. CrossRef Medline

Hoover WB, Vertes RP (2011) Projections of the medial orbital and ventral orbital cortex in the rat. J Comp Neurol 519:3766-3801. CrossRef Medline

Ishii H, Ohara S, Tobler PN, Tsutsui K, Iijima T (2012) Inactivating anterior insular cortex reduces risk taking. J Neurosci 32:16031-16039. CrossRef Medline

Jezzini A, Mazzucato L, La Camera G, Fontanini A (2013) Processing of hedonic and chemosensory features of taste in medial prefrontal and insular networks. J Neurosci 33:18966-18978. CrossRef Medline

King CT, Hashimoto K, Blonde GD, Spector AC (2015) Unconditioned oromotor taste reactivity elicited by sucrose and quinine is unaffected by extensive bilateral damage to the gustatory zone of the insular cortex in rats. Brain Res 1599:9-19. CrossRef Medline

Knapska E, Maren S (2009) Reciprocal patterns of c-Fos expression in the medial prefrontal cortex and amygdala after extinction and renewal of conditioned fear. Learn Mem 16:486-493. CrossRef Medline

Lara AH, Kennerley SW, Wallis JD (2009) Encoding of gustatory working memory by orbitofrontal neurons. J Neurosci 29:765-774. CrossRef Medline

LeDoux JE (2000) Emotion circuits in the brain. Annu Rev Neurosci 23: 155-184. CrossRef Medline

LeDoux JE, Cicchetti P, Xagoraris A, Romanski LM (1990) The lateral amygdaloid nucleus: sensory interface of the amygdala in fear conditioning. J Neurosci 10:1062-1069. Medline

Lin PY, Wang SP, Tai MY, Tsai YF (2010) Differential involvement of medial prefrontal cortex and basolateral amygdala extracellular signalregulated kinase in extinction of conditioned taste aversion is dependent on different intervals of extinction following conditioning. Neuroscience 171:125-133. CrossRef Medline

MacDonald CJ, Meck WH, Simon SA, Nicolelis MA (2009) Taste-guided decisions differentially engage neuronal ensembles across gustatory cortices. J Neurosci 29:11271-11282. CrossRef Medline

Machado CJ, Bachevalier J (2007) The effects of selective amygdala, orbital frontal cortex or hippocampal formation lesions on reward assessment in nonhuman primates. Eur J Neurosci 25:2885-2904. CrossRef Medline

Maren S, Quirk GJ (2004) Neuronal signalling of fear memory. Nat Rev Neurosci 5:844-852. CrossRef Medline

Marotta R, Fenu S, Scheggi S, Vinci S, Rosas M, Falqui A, Gambarana C, De Montis MG, Acquas E (2014) Acquisition and expression of conditioned taste aversion differentially affects extracellular signal regulated kinase and glutamate receptor phosphorylation in rat prefrontal cortex and nucleus accumbens. Front Behav Neurosci 8:153. CrossRef Medline

Mickley GA, Kenmuir CL, Yocom AM, Wellman JA, Biada JM (2005) A role for prefrontal cortex in the extinction of a conditioned taste aversion. Brain Res 1051:176-182. CrossRef Medline

Milad MR, Quirk GJ (2002) Neurons in medial prefrontal cortex signal memory for fear extinction. Nature 420:70-74. CrossRef Medline

Namburi P, Al-Hasani R, Calhoon GG, Bruchas MR, Tye KM (2016) Architectural representation of valence in the limbic system. Neuropsychopharmacology 41:1697-1715. CrossRef Medline

Ostlund SB, Balleine BW (2007) The contribution of orbitofrontal cortex to action selection. Ann N Y Acad Sci 1121:174-192. CrossRef Medline

Padoa-Schioppa C, Assad JA (2008) The representation of economic value in the orbitofrontal cortex is invariant for changes of menu. Nat Neurosci 11:95-102. CrossRef Medline

Parkes SL, Balleine BW (2013) Incentive memory: evidence the basolateral amygdala encodes and the insular cortex retrieves outcome values to guide choice between goal-directed actions. J Neurosci 33:8753-8763. CrossRef Medline

Paulus MP (2007) Decision-making dysfunctions in psychiatry-altered homeostatic processing? Science 318:602-606. CrossRef Medline

Paxinos G, Watson C (1998) The rat brain in stereotaxic coordinates. San Diego: Academic.

Pickens CL, Saddoris MP, Setlow B, Gallagher M, Holland PC, Schoenbaum G (2003) Different roles for orbitofrontal cortex and basolateral amygdala in a reinforcer devaluation task. J Neurosci 23:11078-11084. Medline

Pickens CL, Saddoris MP, Gallagher M, Holland PC (2005) Orbitofrontal lesions impair use of cue-outcome associations in a devaluation task. Behav Neurosci 119:317-322. CrossRef Medline

Price JL (2007) Definition of the orbital cortex in relation to specific connections with limbic and visceral structures and other cortical regions. Ann N Y Acad Sci 1121:54-71. CrossRef Medline

Quirk GJ, Armony JL, LeDoux JE (1997) Fear conditioning enhances different temporal components of tone-evoked spike trains in auditory cortex and lateral amygdala. Neuron 19:613-624. CrossRef Medline

Rangel A, Camerer C, Montague PR (2008) A framework for studying the neurobiology of value-based decision making. Nat Rev Neurosci 9:545556. CrossRef Medline

Rich EL, Wallis JD (2014) Medial-lateral organization of the orbitofrontal cortex. J Cogn Neurosci 26:1347-1362. CrossRef Medline 
Rich EL, Wallis JD (2016) Decoding subjective decisions from orbitofrontal cortex. Nat Neurosci 19:973-980. CrossRef Medline

Rolls ET (2004) The functions of the orbitofrontal cortex. Brain Cogn 55: 11-29. CrossRef Medline

Schoenbaum G, Roesch M (2005) Orbitofrontal cortex, associative learning, and expectancies. Neuron 47:633-636. CrossRef Medline

Schoenbaum G, Setlow B (2001) Integrating orbitofrontal cortex into prefrontal theory: common processing themes across species and subdivisions. Learn Mem 8:134-147. CrossRef Medline

Schoenbaum G, TakahashiY, Liu TL, McDannald MA (2011) Does the orbitofrontal cortex signal value? Ann N Y Acad Sci 1239:87-99. CrossRef Medline

Sierra-Mercado D, Padilla-Coreano N, Quirk GJ (2011) Dissociable roles of prelimbic and infralimbic cortices, ventral hippocampus, and basolateral amygdala in the expression and extinction of conditioned fear. Neuropsychopharmacology 36:529-538. CrossRef Medline

Sotres-Bayon F, Quirk GJ (2010) Prefrontal control of fear: more than just extinction. Curr Opin Neurobiol 20:231-235. CrossRef Medline
Sotres-Bayon F, Diaz-Mataix L, Bush DE, LeDoux JE (2009) Dissociable roles for the ventromedial prefrontal cortex and amygdala in fear extinction: NR2B contribution. Cereb Cortex 19:474-482. CrossRef Medline

Stalnaker TA, Cooch NK, Schoenbaum G (2015) What the orbitofrontal cortex does not do. Nat Neurosci 18:620-627. CrossRef Medline

Sugrue LP, Corrado GS, Newsome WT (2005) Choosing the greater of two goods: neural currencies for valuation and decision making. Nat Rev Neurosci 6:363-375. CrossRef Medline

Tremblay L, Schultz W (1999) Relative reward preference in primate orbitofrontal cortex. Nature 398:704-708. CrossRef Medline

Wallis JD (2012) Cross-species studies of orbitofrontal cortex and valuebased decision-making. Nat Neurosci 15:13-19. CrossRef Medline

Xin J, Ma L, Zhang TY, Yu H, Wang Y, Kong L, Chen ZY (2014) Involvement of BDNF signaling transmission from basolateral amygdala to infralimbic prefrontal cortex in conditioned taste aversion extinction. J Neurosci 34:7302-7313. CrossRef Medline 